\title{
Muscle mitochondria and insulin resistance: a human perspective.
}

Citation for published version (APA):

Hoeks, J., \& Schrauwen, P. (2012). Muscle mitochondria and insulin resistance: a human perspective. Trends in Endocrinology and Metabolism, 23(9), 444-450. https://doi.org/10.1016/j.tem.2012.05.007

Document status and date:

Published: 01/09/2012

DOI:

10.1016/j.tem.2012.05.007

Document Version:

Publisher's PDF, also known as Version of record

Document license:

Taverne

Please check the document version of this publication:

- A submitted manuscript is the version of the article upon submission and before peer-review. There can be important differences between the submitted version and the official published version of record.

People interested in the research are advised to contact the author for the final version of the publication, or visit the DOI to the publisher's website.

- The final author version and the galley proof are versions of the publication after peer review.

- The final published version features the final layout of the paper including the volume, issue and page numbers.

Link to publication

\footnotetext{
General rights rights.

- You may freely distribute the URL identifying the publication in the public portal. please follow below link for the End User Agreement:

www.umlib.nl/taverne-license

Take down policy

If you believe that this document breaches copyright please contact us at:

repository@maastrichtuniversity.nl

providing details and we will investigate your claim.
}

Copyright and moral rights for the publications made accessible in the public portal are retained by the authors and/or other copyright owners and it is a condition of accessing publications that users recognise and abide by the legal requirements associated with these

- Users may download and print one copy of any publication from the public portal for the purpose of private study or research.

- You may not further distribute the material or use it for any profit-making activity or commercial gain

If the publication is distributed under the terms of Article $25 \mathrm{fa}$ of the Dutch Copyright Act, indicated by the "Taverne" license above, 


\title{
Special Issue: The evolving role of mitochondria in metabolism Muscle mitochondria and insulin resistance: a human perspective
}

\author{
Joris Hoeks and Patrick Schrauwen
}

NUTRIM - School for Nutrition, Toxicology and Metabolism, Department of Human Biology, Maastricht University Medical Center, 6200 MD Maastricht, The Netherlands

\begin{abstract}
Reduced mitochondrial capacity in skeletal muscle has been suggested to underlie the development of insulin resistance and type 2 diabetes mellitus (T2DM). However, data obtained from human subjects concerning this putative relation indicate that the mitochondrial defect observed in diabetic muscle might be secondary to the insulin-resistant state instead of being a causal factor. Nonetheless, diminished mitochondrial function, even secondary to insulin resistance, may accelerate lipid deposition in non-adipose tissues and aggravate insulin resistance. Indeed, improving mitochondrial capacity via exercise training and calorie restriction is associated with positive metabolic health effects. Here we review muscle mitochondrial dysfunction in humans and propose that targeting muscle mitochondria to improve muscle oxidative capacity should be considered as a strategy for improving metabolic health.
\end{abstract}

\section{Introduction}

The prevalence of diabetes mellitus is reaching epidemic proportions and the number of people suffering from this disease will roughly double between the year 2000 and 2030 , from 171 to 366 million patients, respectively [1]. The tremendous number of the current and predicted future prevalence of diabetes mellitus worldwide is paralleled by a similar increase in the prevalence of overweight and obesity. Obesity is an important risk factor for T2DM and insulin resistance, an early feature in the pathogenesis of T2DM characterized by the relative inability of the hormone insulin to induce glucose uptake in peripheral tissues. Because skeletal muscle accounts for $\sim 80 \%$ of the insulin-stimulated disposal of postprandial glucose [2], this tissue is considered a major player in the development of insulin resistance and T2DM.

Overconsumption of high-energy nutrients (especially fats) and reduced level of physical activity lead to an expansion of the adipose tissue and obesity, but may also result in fat accumulation in non-adipose tissues, such as skeletal muscle but also liver and heart [3-5]. Because muscle fat accumulation is negatively correlated with insulin sensitivity [6,7], intramuscular fat levels provide a likely link between obesity and the development of T2DM and the multiple putative mechanisms involved are currently under investigation.

Corresponding author: Schrauwen, P. (p.schrauwen@maastrichtuniversity.nl). Keywords: mitochondrial capacity; calorie restriction; diabetes; resveratrol.
In essence, fat accumulation in skeletal muscle tissue results from an imbalance between the supply and the oxidation of fatty acids, resulting in the storage of fatty acids as intramuscular triacylglycerols (IMTG). In that respect, it has been shown that insulin-resistant subjects and T2DM patients are characterized by a decreased capacity to oxidize fatty acids $[8,9]$. Although earlier reports already indicated that the activity of oxidative enzymes in skeletal muscle was reduced in T2DM patients [10,11], it was however not until recently that the interest in muscle mitochondrial (dys)function and the development of T2DM began to expand.

In the present review we provide an overview of the studies that directly address the relation between skeletal muscle mitochondrial function and the development of insulin resistance and T2DM. Although a large body of data on this topic has been collected in animal and cell studies, here we focus solely on studies performed in humans, including primary human muscle cells.

\section{Mitochondrial dysfunction and insulin resistance Human studies linking mitochondrial dysfunction to insulin resistance}

Interest in the relation between reduced muscle mitochondrial function (Box 1) and insulin resistance exploded about a decade ago when, in 2002, Kelley et al. described dysfunctional skeletal muscle mitochondria in T2DM subjects, exemplified by reduced activity of the mitochondrial enzymes NADH: $\mathrm{O}_{2}$ reductase and citrate synthase $(\mathrm{CS})$ as well as by aberrant mitochondrial morphology assessed by electron microscopy [12]. In addition, two influential papers identified a coordinated reduction in the expression of oxidative genes in skeletal muscle tissue of T2DM patients, as well as in insulin-resistant subjects with a positive family history for T2DM $[13,14]$. These studies also showed that the peroxisome proliferator-activated receptor $\gamma$ coactivator $1 \alpha$ (PGC- $1 \alpha)$, a crucial regulator of mitochondrial metabolism, was reduced in T2DM patients $[13,14]$. These findings were of importance because the lowered oxidative gene expression pattern was not only seen in T2DM, but also in 'pre-diabetic' subjects (insulinresistant offspring of T2DM patients), indicating that this might be a primary event in the pathogenesis of T2DM [14]. Furthermore, with the lowered PGC-1 $\alpha$ levels, a possible molecular mechanism was also identified. Thus, lower PGC-1 $\alpha$ levels, possibly due to genetic predisposition, inactivity, or excess nutrient intake, would lead to a 


\section{Box 1. Parameters of muscle oxidative capacity}

Total oxidative or ATP-producing capacity of a muscle is determined by the number of mitochondria present in the muscle (mitochondrial density), the functional capacity per mitochondrion (intrinsic mitochondrial function), and by muscle perfusion and thus oxygen and substrate supply. Non-invasive magnetic resonance spectroscopy (MRS) has been used to estimate in vivo skeletal muscle mitochondrial oxidative capacity. A saturation transfer method has been used to determine the unidirectional flux through ATP synthase (ATP synthetic flux, fATPase) in the non-exercising state, by temporarily suppressing the signal from ATP and measuring the subsequent changes in free phosphate to calculate unidirectional ATP synthesis rate. Simultaneously, flux through the tricarboxylic acid (TCA or Krebs) cycle can be determined non-invasively by carbon-13 $\left({ }^{13} \mathrm{C}\right)$ MRS. An alternative method measures the resynthesis rate of phosphocreatine ( $\mathrm{PCr}$ ), an important energy-buffering system, during exercise. $\mathrm{PCr}$ decreases during exercise and is rapidly recovered postexercise, the latter being driven by mitochondrial ATP production. $\mathrm{PCr}$ recovery after exercise is therefore an indirect measure of mitochondrial oxidative capacity. In contrast to the magnetization saturation transfer method, the PCR-recovery methodology thus assesses oxidative capacity under conditions of increased metabolic demand.

Mitochondrial function can also be determined ex vivo in biopsy material. The intrinsic mitochondrial capacity, in other words the oxidative capacity per mitochondrion, is commonly obtained by measuring ex vivo oxygen consumption upon various experimental conditions in isolated skeletal mitochondria or in permeabilized muscle fibers, corrected for mitochondrial density. Next to function, mitochondrial density can be estimated by simply counting mitochondria in electron microscopy (EM) pictures, by measurement of mitochondrial DNA copy number relative to the nuclear DNA (assuming that the amount of mitochondrial DNA copies per mitochondrion is invariable), or by determination of mitochondria marker protein levels and/or activity, such as the commonly assessed TCA cycle enzyme citrate synthase (CS).

Importantly, a clear definition of mitochondrial dysfunction is lacking, and may therefore reflect reductions in any of the abovementioned parameters.

reduced generation of mitochondrial proteins and most likely a loss of functional capacity [14]. This concept was underscored by the finding that, in women with polycystic ovary syndrome (PCOS), who are characterized by muscle insulin resistance, there was reduced expression of genes involved in mitochondrial metabolism, possibly accounted for by reduced PGC- $1 \alpha$ expression [15]. A later report using a proteomics analysis extended the reduction of oxidative gene expression to the protein level, showing patterns of reduced abundance of mitochondrial proteins in skeletal muscle from T2DM patients [16].

These studies demonstrated that insulin resistance is associated with a reduction in mitochondrial protein levels, probably as a result of reduced PGC- $1 \alpha$ expression. Theoretically, this would result in reduced skeletal muscle oxidative capacity. To address this hypothesis, functional approaches using in vivo non-invasive magnetic resonance spectroscopy (MRS) were performed. Indeed, lean insulinresistant elderly subjects showed a $40 \%$ reduction in both resting muscle tricarboxylic acid (TCA) cycle flux, and ATP synthesis rate, when assessed by ${ }^{13} \mathrm{C}$ and ${ }^{31} \mathrm{P}$ MRS (Box 1), respectively [17]. In healthy lean insulin-resistant offspring of T2DM patients, a $\sim 30 \%$ reduction in resting ATP synthesis rate, as assessed by ${ }^{31} \mathrm{P}$ MRS, was observed [18]. This reduction was associated with an $\sim 80 \%$ increase in muscle lipid accumulation which the authors attributed to reduced mitochondrial activity, thereby further position- ing mitochondrial dysfunction as a primary cause for muscle insulin resistance [18]. Further support was obtained in another study of insulin-resistant T2DM offspring; here the authors, using similar MRS methodologies, reported a substantial $(\sim 30 \%)$ reduction in resting muscle TCA flux associated with an increase in muscle lipid content [19]. Others have confirmed the impaired muscle oxidative capacity in T2DM patients, although in a later study that used ${ }^{31} \mathrm{P}$ MRS, this was demonstrated as a slower phosphocreatine (PCr) recovery rate (Box 1$)$ after exercise [20].

Although the reduced muscle oxidative capacity, assessed by these various MRS methods, will be largely accounted for by the amount of mitochondria present in the muscle as well as the intrinsic mitochondrial capacity, in other words, the functional capacity per mitochondrion, other factors - such as perfusion and muscle fiber type composition - may also be involved. Therefore, to further prove that mitochondrial (dys)function is associated with insulin resistance, intrinsic mitochondrial function and mitochondrial density were extensively studied in the context of insulin resistance and T2DM.

Mitochondrial DNA (mtDNA) content, frequently used as a marker for mitochondrial density (Box 1), was shown to be lower in T2DM patients, in comparison to lean controls, but similar to that of obese subjects [21]. Morino et al. [22] found that mitochondrial density, assessed by electron microscopy, was reduced by $\sim 38 \%$ in young lean, insulinresistant T2DM offspring versus insulin-sensitive controls, matched for age, body mass index (BMI) and physical activity. At the same time, insulin-stimulated glucose uptake was decreased and IMTG levels were increased by $\sim 60 \%$ [22]. By contrast, it has been shown that mtDNA copy number was not different between T2DM patients, insulin-resistant first-degree relatives of T2DM patients, and age- and BMI-matched controls [23]. In skeletal muscle, mitochondria can be found both at the sarcolemma and located intermyofibrillary. Ritov et al. [21] reported a decrease specifically in subsarcolemmal mitochondria in T2DM patients compared to lean and obese controls. By contrast, Chomentowski et al. [24] found a similar content of subsarcolemmal mitochondria in lean insulin-sensitive controls, insulin-resistant nondiabetic subjects, and T2DM patients, whereas intermyofibrillar mitochondrial content was lower in the insulin-resistant nondiabetic subjects and T2DM patients versus lean controls. Furthermore, the lower intermyofibrillar mitochondrial content correlated significantly with fasting respiratory quotient and metabolic flexibility [24]. In line with the latter finding, it was shown previously that mtDNA content is reduced in individuals with a family history of diabetes and correlates positively with metabolic flexibility [25].

Several studies also aimed to test whether intrinsic mitochondrial defects are present in T2DM patients. In this context, Ritov et al. [21] showed a decrease in overall activity of the electron transport chain (ETC) in T2DM patients versus lean (but not versus obese) controls, that could not be fully explained by the observed decrease in mtDNA, suggesting an additional intrinsic impairment of mitochondrial function. Furthermore, although the overall ETC capacity in T2DM patients was similar to obese controls, 
subsarcolemmal - but not intermyofibrillar - mitochondrial fractions displayed reduced ETC activity in T2DM patients versus obese subjects [21]. In further support for an intrinsic mitochondrial defect in T2DM, it was reported that skeletal muscle mitochondria isolated from T2DM patients show a small but significant reduction in mitochondrial respiration rate per mitochondrion [26]. Mitochondrial respiration can also be measured ex vivo in muscle fiber bundles that are carefully separated and subsequently permeabilized by saponin, thereby allowing the entry of substrates and stimulators to assess mitochondrial function, while leaving the surrounding cell relatively intact. Using this approach, oxygen consumption rates in permeabilized muscle fibers were significantly reduced in T2DM patients, but this difference disappeared when the oxygen flux was normalized for mitochondrial DNA content. This finding indicated that the blunted mitochondrial respiration in T2DM muscle could be fully attributed to a lower mitochondrial content rather than to an intrinsic defect [27]. This result was later reproduced in two other studies $[28,29]$. By contrast, it has been previously shown that mitochondrial respiration in permeabilized muscle fibers, when corrected for mtDNA copy number, was $\sim 35 \%$ lower in diabetic patients, compared to BMI-matched control subjects, with intermediate values for first-degree relatives of T2DM patients [23]. This difference in intrinsic mitochondrial function between T2DM patients and BMI-matched controls was also confirmed in a later study [30].

In an attempt to distinguish between genetic and environmental factors, Minet et al. [31] employed human cultured primary myotubes from lean, obese, and T2DM donors to isolate and study mitochondria. Although this study did not reveal a difference in mitochondrial content between the groups, the dynamic equilibrium between physiological ATP synthesis and ATP consumption rates (mimicked by the addition of the ATP-consuming enzyme hexokinase) was $\sim 35 \%$ lower in myotubes derived from T2DM patients compared to lean controls [31,32]. The authors proposed that the mitochondrial defect seen in T2DM patients is due to a primarily impaired ATP synthesis and an adaptive loss of mitochondrial mass [31,32].

Taken together, the reports describing an impairment of muscle mitochondrial metabolism in T2DM and insulin resistance are numerous, although these reports fail to elucidate fully whether the functional defect should be ascribed to changes in mitochondrial content (of distinct pools), to intrinsic mitochondrial capacity, or to both.

\section{Human studies disconnecting mitochondrial dysfunction and insulin resistance}

Although many reports support the presence of a mitochondrial defect in T2DM and insulin resistance, it is arguable whether the associative nature of virtually all studies allows us to conclude that there is a cause-andeffect relation [33,34]. In this context, it has also been shown that prolonged $(60 \mathrm{~h})$ fasting in healthy young individuals results in an elevation of intramuscular lipid levels, pronounced insulin resistance, and reduced intrinsic mitochondrial capacity, despite a marked increase in whole-body fat oxidation [35]. This is in contrast with the concept that mitochondrial dysfunction is a primary defect that would lead to a decreased fat oxidative capacity and intramuscular fat accumulation, resulting in insulin resistance. Moreover, many other studies have shown a disconnection between muscle mitochondrial function and insulin sensitivity, arguing against the primary nature of the mitochondrial defect in the pathogenesis of T2DM.

In this context, insulin is a potent stimulator of mitochondrial metabolism, which led to the belief that the mitochondrial dysfunction observed in T2DM might be secondary to the insulin-resistant state, and not a causal player. Indeed, it was shown that insulin-stimulated mitochondrial ATP synthesis (determined by ${ }^{31} \mathrm{P}$ MRS) severely blunted the muscle from T2DM patients [36] and from insulin-resistant offspring of T2DM patients [37]. In addition, muscle mitochondrial protein synthesis was responsive to insulin in nondiabetic individuals [36], whereas insulin failed to promote mitochondrial protein synthesis in T2DM patients [38]. Asmann et al. [39] compared T2DM patients with age- and BMI-matched controls for several mitochondrial parameters under conditions of low versus high circulating insulin levels, and found a reduced mitochondrial ATP production rate in T2DM patients, but only in association with high circulating insulin [39]. This blunted response in mitochondrial ATP production was accompanied by reduced glucose disposal and diminished expression of several genes key to mitochondrial metabolism (CS, PGC-1 $\alpha$ and cytochrome $c$ oxidase), suggesting that mitochondrial dysfunction in T2DM is a secondary defect related to the impaired response to insulin [39].

Furthermore, in cultured human skeletal muscle cells, impaired cellular respiratory function - induced by the addition of the mitochondrial inhibitor sodium azide - was without effect on insulin signaling or insulin-stimulated glucose uptake [40]. Another study demonstrated that nondiabetic Americans of Northern European descent were more insulin-sensitive than either diabetic or nondiabetic Asian Indians, despite lower skeletal muscle mitochondrial oxidative phosphorylation (OxPhos) capacity (i.e., lower mtDNA copy number), mRNA of OxPhos genes, and CS activity [41]. Moreover, diabetic and nondiabetic Asian Indians differed substantially in insulin sensitivity, but skeletal muscle OxPhos capacity was similar [41]. In addition, several studies were performed to determine whether interventions with beneficial effects on wholebody insulin sensitivity were paralleled by improvements in skeletal muscle mitochondrial function. Toledo et al. [42] compared the effects of weight loss induced by either diet alone or by diet combined with exercise, in sedentary overweight/obese individuals, and found that both interventions led to similar improvements in body weight and insulin sensitivity. Despite the advantageous metabolic effects upon both interventions, only the combined dietexercise intervention led to increased mitochondrial content (assessed by electron microscopy) as well as increased ETC activity; diet alone did not affect mitochondrial function [42]. Treating T2DM patients for eight weeks with the insulin-sensitizing agent rosiglitazone, which significantly improves insulin sensitivity, also was without effect on in vivo mitochondrial function as determined by post-exercise PCr recovery half-time [43]. Similar findings were obtained 
in another study in which a 12 week rosiglitazone treatment in T2DM patients improved glycemic control but did not augment maximal oxygen consumption $\left(\mathrm{VO}_{2 \max }\right)$ or skeletal muscle mtDNA copy-number [44].

Together these findings indicate that the insulin-sensitizing effects of diet-induced weight loss and rosiglitazone treatment occur in the absence of an improvement in skeletal muscle mitochondrial capacity. Although this does not exclude mitochondrial dysfunction as a contributing factor, these findings argue against mitochondrial dysfunction as the primary culprit in the development of insulin resistance and T2DM. This notion is further substantiated by findings in young lean men born with low birth-weight, who displayed several pre-diabetic characteristics but normal in vivo mitochondrial function $\left({ }^{31} \mathrm{P} \mathrm{MRS}\right)$ and normal expression of OxPhos genes [45]. Furthermore, primary myotubes cultured from women with PCOS, characterized by insulin resistance, displayed normal mitochondrial mass and mitochondrial ATP synthesis in comparison to myotubes cultured from weight- and age-matched controls [46]. Therefore, it was suggested that the earlier observed decrease in the expression of OxPhos genes in these subjects [15] is an adaptive trait rather than a primary defect [46]. Also the coordinated reduction in the expression of oxidative genes that was previously reported in T2DM patients [13,14], has been challenged by others. Thus, Gallagher et al. [47] studied a much larger cohort of T2DM patients versus controls, matched for several potential confounders, and found no evidence for a defect in the expression of oxidative genes in skeletal muscle of T2DM patients. Finally, in patients with genetic defects in insulin-receptor signaling, in vivo mitochondrial function, determined as PCr recovery after exercise by ${ }^{31} \mathrm{P}$ MRS, was significantly slower compared to healthy age-, fitness-, and BMI-matched controls, suggesting that impairments in insulin signaling may promote mitochondrial dysfunction [48], instead of the other way around.

In summary, there is limited evidence to support the concept that the skeletal muscle mitochondrial dysfunction seen in human insulin resistance is the cause of T2DM, and this is underscored by the fact that improved insulin sensitivity does not require changes in skeletal muscle mitochondrial function. However, the presence of a mitochondrial defect in insulin resistance and/or T2DM, even if secondary to the insulin-resistant state, is likely to accelerate ectopic fat accumulation and exacerbate insulin resistance. Therefore, the question that remains to be answered is whether targeting muscle mitochondria to improve muscle oxidative capacity could be considered a successful strategy to improve metabolic health.

\section{Therapeutic potential of enhancing muscle mitochondrial capacity}

\section{Aerobic exercise training in insulin resistance}

Irrespective of the presence or absence of a prominent mitochondrial defect in skeletal muscle of insulin-resistant subject and T2DM patients, regular exercise training, which enhances muscle oxidative capacity, is accompanied by pronounced and beneficial effects on insulin sensitivity and other markers of metabolic health. Indeed, 10 week aerobic cycling training in obese, male T2DM patients versus age- and BMI-matched controls induced a 20\% increase in insulin sensitivity in both groups, which was associated with a $\sim 40 \%$ increase in mitochondrial content in both groups [49]. In addition, it has been shown that a 12 week combined progressive training program relieved the impairment of in vivo mitochondrial function in T2DM patients [30]. The reduced intrinsic mitochondrial capacity was also confirmed in a subset of these patients [23], and mitochondrial respiration in permeabilized muscle fibers was found to be improved significantly and restored to control levels after the 12 week training program. These improvements in mitochondrial function were mainly accounted for by an increase in mitochondrial density, as exemplified by mtDNA copy number [50]. In T2DM patients, improvement in skeletal muscle mitochondrial function was paralleled by a significant improvement in insulin sensitivity which, however, was not restored to control levels, again underscoring that mitochondrial dysfunction cannot completely explain lower muscle insulin sensitivity in T2DM [50].

Finally, Hey-Mogensen et al. [51] studied isolated skeletal muscle mitochondria from male T2DM patients versus age-, BMI-, and physical activity-matched controls, both before and after a 10 week aerobic training program. Training resulted in a significant increase in mitochondrial respiratory capacity per $\mathrm{kg}$ of muscle mass. In addition, intrinsic mitochondrial capacity (i.e., mitochondrial respiration corrected for CS activity) was improved without showing a difference in training adaptability between T2DM patients and controls [51]. Again, these adaptations in mitochondrial metabolism were associated with enhanced insulin sensitivity, both in T2DM patients $(+13 \%)$ and in controls $(+22 \%)$ [51].

Taken together, it is evident that moderate (combined) aerobic exercise training has beneficial effects on mitochondrial metabolism, and is associated with improved insulin sensitivity. However, additional human studies are required to conclude if the increase in mitochondrial function in humans is causally related to the improvement in insulin sensitivity.

\section{Calorie restriction, resveratrol, and metabolic health}

In addition to exercise training, calorie restriction has also been shown to enhance mitochondrial oxidative capacity in human skeletal muscle and has been associated with positive effects on metabolic health. Indeed, six months of $25 \%$ calorie restriction in young overweight subjects resulted in reductions in fat mass, intrahepatic lipid content, serum lipid levels, fasting insulin levels, and other markers for insulin sensitivity [52-54]. This improved metabolic profile was accompanied by beneficial effects on muscle oxidative capacity, exemplified by an increased expression of PGC- $1 \alpha$, mitochondrial transcription factor A (TFAM) and sirtuin 1 (SIRT1), and a 35\% increase in mtDNA content in skeletal muscle tissue [55].

Although the data are derived from animal and cell studies, a growing body of evidence indicates that SIRT1, the founding member of the sirtuin protein family of NAD-dependent deacetylases, may be the key molecule that regulates mitochondrial function, energy homeostasis, and insulin sensitivity, also in response to calorie 
restriction (reviewed in [56]). Although data for humans are surprisingly scarce, the expression of SIRT1 has also been linked to insulin resistance and T2DM; in peripheral blood mononuclear cells (PBMCs), insulin resistance and metabolic syndrome were associated with low SIRT1 gene and protein expression [57]. Furthermore, it was also shown that low SIRT1 expression in human adipose tissue might reflect the impaired regulation of mitochondrial function associated with insulin resistance [58]. In addition, Fröjdö et al. [59] reported decreased SIRT1 protein levels in muscle biopsies and primary myotubes derived from T2DM patients, suggesting a contribution of diminished SIRT1 in the determination of muscle insulin resistance.

Although exercise training and calorie restriction are highly effective in increasing muscle oxidative capacity and improving metabolic health, most people can have difficulties in adhering to a strict diet or exercise regimens. Therefore, in recent years a search for other stimulators of the AMPK-SIRT1-PGC1 $\alpha$ axis (Figure 1) to enhance mitochondrial oxidative capacity has been initiated.

In this context, resveratrol, a natural polyphenolic small-molecule compound, was discovered to activate SIRT1, most likely via activation of AMPK. Recently, the effect of resveratrol supplementation on mitochondrial and energy metabolism and on markers of insulin sensitivity was examined in human volunteers. It was shown that 30 days of resveratrol supplementation, in healthy but obese subjects, activated skeletal muscle AMPK and increased the protein levels of both SIRT1 and PGC- $1 \alpha$, similar to findings in animal studies [60]. Furthermore, CS activity was enhanced without change in mtDNA copy number, and mitochondrial respiration in permeabilized muscle fibers was increased [60]. The adaptations were paralleled by an improved homeostatic model assessment (HOMA) index and reduced hepatic lipid levels [60]. In fact, resveratrol supplementation mimicked in many aspects the effects observed in humans after calorie restriction.

In summary, improving mitochondrial function via calorie restriction and resveratrol supplementation has very promising beneficial effects on metabolic health (Figure 1). Whether or not resveratrol is able to alleviate metabolic mitochondrial aberrations characterizing T2DM remains to be elucidated.

\section{Concluding remarks}

A clear evaluation of the causal relation between muscle mitochondrial function and insulin resistance in humans is hindered by the many diverse definitions of mitochondrial dysfunction as well as the various methods and techniques available to determine muscle oxidative capacity and/or mitochondrial function. However, based on the currently available human data reviewed in this paper, we are inclined to believe that skeletal muscle mitochondrial dysfunction is not a primary defect in the development of insulin resistance. Nonetheless, improving muscle oxidative capacity and mitochondrial function by exercise training, calorie restriction, or resveratrol supplementation has been associated with improved metabolic health in humans. Future research should be devoted to the optimization of the exercise-training interventions and to testing the efficacy of resveratrol in T2DM patients, especially in the long-term. Furthermore, it will be of great importance to identify additional activators of AMPK, SIRT1, or PGC-1 $\alpha$, and other stimulators of muscle mitochondrial capacity, because

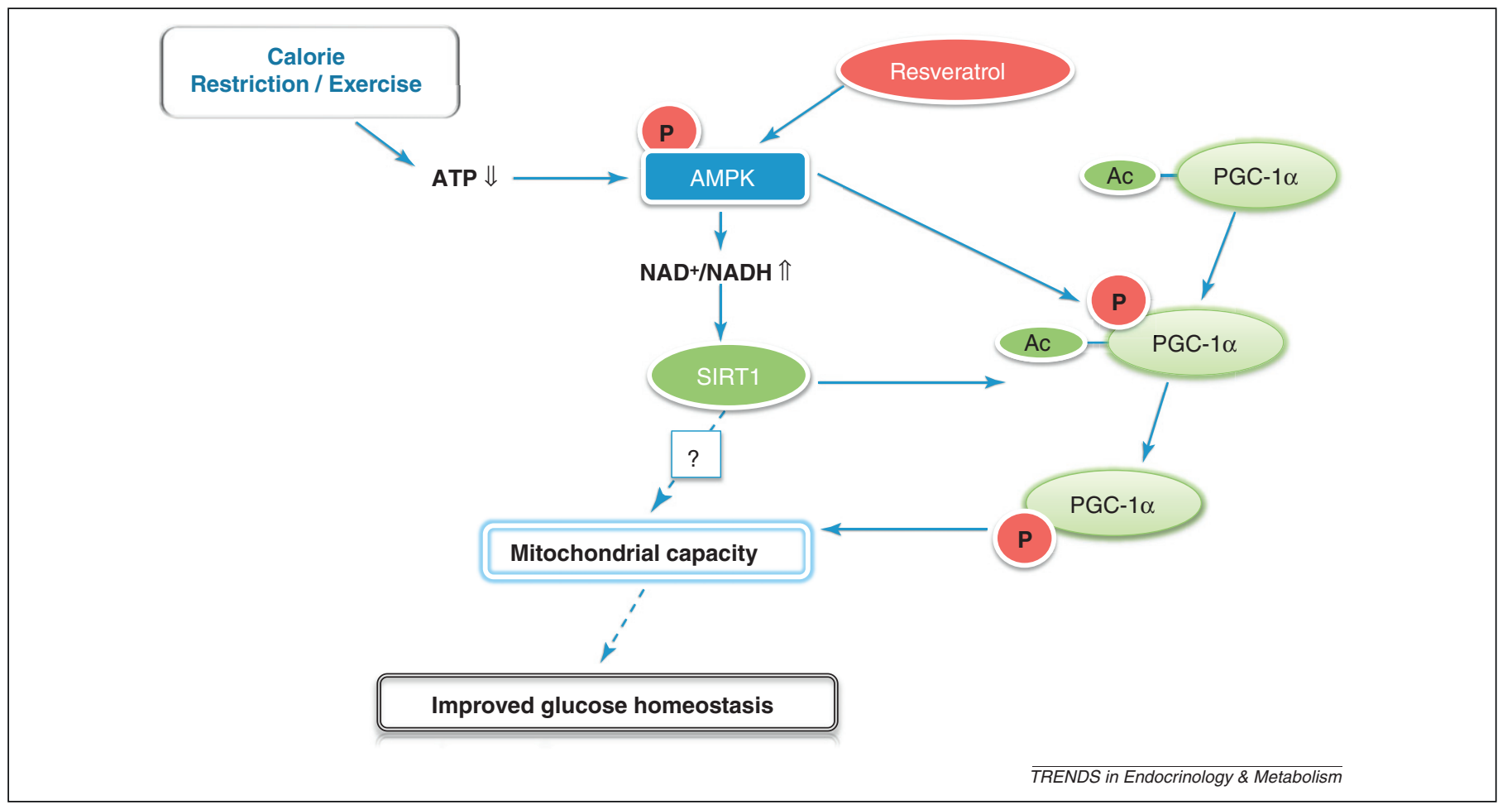

Figure 1. Molecular pathways involved in the beneficial effects of calorie restriction, exercise, and resveratrol on mitochondrial function and metabolic health. Phosphorylation and activation of AMPK upon reduced ATP levels (due to calorie restriction or exercise), or directly by resveratrol, triggers an increase in the cellular NAD $/ \mathrm{NADH}$ ratio, which activates sirtuin 1 (SIRT1). In addition, AMPK also phosphorylates peroxisome proliferator-activated receptor $\gamma$ coactivator $1 \alpha$ (PGC-1 $\alpha$ ) and primes it for subsequent deacetylation by SIRT1. The impact of AMPK and SIRT1 on the acetylation status of PGC-1 $\alpha$, and possibly of other transcriptional regulators, subsequently improves mitochondrial capacity and metabolic health (adapted from [61]). 
these are likely to offer a promising new strategy for improving the metabolic profiles of insulin-resistant subjects and T2DM patients.

\section{References}

1 Wild, S. et al. (2004) Global prevalence of diabetes: estimates for the year 2000 and projections for 2030. Diabetes Care 27, 1047-1053

2 Shulman, G.I. et al. (1990) Quantitation of muscle glycogen synthesis in normal subjects and subjects with non-insulin-dependent diabetes by ${ }^{13} \mathrm{C}$ nuclear magnetic resonance spectroscopy. N. Engl. J. Med. 322, 223-228

3 Bachmann, O.P. et al. (2001) Effects of intravenous and dietary lipid challenge on intramyocellular lipid content and the relation with insulin sensitivity in humans. Diabetes 50, 2579-2584

4 Schrauwen-Hinderling, V.B. et al. (2005) Intramyocellular lipid content and molecular adaptations in response to a 1-week high-fat diet. Obes. Res. 13, 2088-2094

5 van Herpen, N.A. et al. (2011) Three weeks on a high-fat diet increases intrahepatic lipid accumulation and decreases metabolic flexibility in healthy overweight men. J. Clin. Endocrinol. Metab. 96, E691-E695

$6 \mathrm{Krssak}$, M. et al. (1999) Intramyocellular lipid concentrations are correlated with insulin sensitivity in humans: a ${ }^{1} \mathrm{H}$ NMR spectroscopy study. Diabetologia 42, 113-116

7 Perseghin, G. et al. (1999) Intramyocellular triglyceride content is a determinant of in vivo insulin resistance in humans: a ${ }^{1} \mathrm{H}-{ }^{13} \mathrm{C}$ nuclear magnetic resonance spectroscopy assessment in offspring of type 2 diabetic parents. Diabetes 48, 1600-1606

8 Blaak, E.E. et al. (2000) Impaired oxidation of plasma-derived fatty acids in type 2 diabetic subjects during moderate-intensity exercise. Diabetes 49, 2102-2107

9 Mensink, M. et al. (2001) Plasma free fatty acid uptake and oxidation are already diminished in subjects at high risk for developing type 2 diabetes. Diabetes 50, 2548-2554

10 Simoneau, J.A. et al. (1999) Markers of capacity to utilize fatty acids in human skeletal muscle: relation to insulin resistance and obesity and effects of weight loss. FASEB J. 13, 2051-2060

11 Simoneau, J.A. and Kelley, D.E. (1997) Altered glycolytic and oxidative capacities of skeletal muscle contribute to insulin resistance in NIDDM. J. Appl. Physiol. 83, 166-171

12 Kelley, D.E. et al. (2002) Dysfunction of mitochondria in human skeletal muscle in type 2 diabetes. Diabetes 51, 2944-2950

13 Mootha, V.K. et al. (2003) PGC-1alpha-responsive genes involved in oxidative phosphorylation are coordinately downregulated in human diabetes. Nat. Genet. 34, 267-273

14 Patti, M.E. et al. (2003) Coordinated reduction of genes of oxidative metabolism in humans with insulin resistance and diabetes: potential role of PGC1 and NRF1. Proc. Natl. Acad. Sci. U.S.A. $100,8466-8471$

15 Skov, V. et al. (2007) Reduced expression of nuclear-encoded genes involved in mitochondrial oxidative metabolism in skeletal muscle of insulin-resistant women with polycystic ovary syndrome. Diabetes 56 , 2349-2355

16 Hwang, H. et al. (2010) Proteomics analysis of human skeletal muscle reveals novel abnormalities in obesity and type 2 diabetes. Diabetes 59 , $33-42$

17 Petersen, K.F. et al. (2003) Mitochondrial dysfunction in the elderly: possible role in insulin resistance. Science 300, 1140-1142

18 Petersen, K.F. et al. (2004) Impaired mitochondrial activity in the insulin-resistant offspring of patients with type 2 diabetes. N. Engl. J. Med. 350, 664-671

19 Befroy, D.E. et al. (2007) Impaired mitochondrial substrate oxidation in muscle of insulin-resistant offspring of type 2 diabetic patients. Diabetes 56, 1376-1381

20 Schrauwen-Hinderling, V.B. et al. (2007) Impaired in vivo mitochondrial function but similar intramyocellular lipid content in patients with type 2 diabetes mellitus and BMI-matched control subjects. Diabetologia 50, 113-120

21 Ritov, V.B. et al. (2005) Deficiency of subsarcolemmal mitochondria in obesity and type 2 diabetes. Diabetes $54,8-14$

22 Morino, K. et al. (2005) Reduced mitochondrial density and increased IRS-1 serine phosphorylation in muscle of insulin-resistant offspring of type 2 diabetic parents. J. Clin. Invest. 115, 3587-3593
23 Phielix, E. et al. (2008) Lower intrinsic ADP-stimulated mitochondrial respiration underlies in vivo mitochondrial dysfunction in muscle of male type 2 diabetic patients. Diabetes 57, 2943-2949

24 Chomentowski, P. et al. (2011) Skeletal muscle mitochondria in insulin resistance: differences in intermyofibrillar versus subsarcolemmal subpopulations and relationship to metabolic flexibility. J. Clin. Endocrinol. Metab. 96, 494-503

25 Ukropcova, B. et al. (2007) Family history of diabetes links impaired substrate switching and reduced mitochondrial content in skeletal muscle. Diabetes 56, 720-727

26 Mogensen, M. et al. (2007) Mitochondrial respiration is decreased in skeletal muscle of patients with type 2 diabetes. Diabetes 56, 1592-1599

27 Boushel, R. et al. (2007) Patients with type 2 diabetes have normal mitochondrial function in skeletal muscle. Diabetologia 50, 790-796

28 Larsen, S. et al. (2011) Increased mitochondrial substrate sensitivity in skeletal muscle of patients with type 2 diabetes. Diabetologia 54 , 1427-1436

29 Rabol, R. et al. (2010) Regional anatomic differences in skeletal muscle mitochondrial respiration in type 2 diabetes and obesity. J. Clin. Endocrinol. Metab. 95, 857-863

30 Meex, R.C. et al. (2010) Restoration of muscle mitochondrial function and metabolic flexibility in type 2 diabetes by exercise training is paralleled by increased myocellular fat storage and improved insulin sensitivity. Diabetes 59, 572-579

31 Minet, A.D. and Gaster, M. (2010) ATP synthesis is impaired in isolated mitochondria from myotubes established from type 2 diabetic subjects. Biochem. Biophys. Res. Commun. 402, 70-74

32 Minet, A.D. and Gaster, M. (2011) The dynamic equilibrium between ATP synthesis and ATP consumption is lower in isolated mitochondria from myotubes established from type 2 diabetic subjects compared to lean control. Biochem. Biophys. Res. Commun. 409, 591-595

33 Raubenheimer, P.J. (2004) Impaired mitochondrial activity and insulin-resistant offspring of patients with type 2 diabetes. N. Engl. J. Med. 350, 2419-2421

34 Short, K.R. et al. (2004) Impaired mitochondrial activity and insulinresistant offspring of patients with type 2 diabetes. N. Engl. J. Med. $350,2419-2421$

35 Hoeks, J. et al. (2010) Prolonged fasting identifies skeletal muscle mitochondrial dysfunction as consequence rather than cause of human insulin resistance. Diabetes 59, 2117-2125

36 Stump, C.S. et al. (2003) Effect of insulin on human skeletal muscle mitochondrial ATP production, protein synthesis, and mRNA transcripts. Proc. Natl. Acad. Sci U.S.A. 100, 7996-8001

37 Petersen, K.F. et al. (2005) Decreased insulin-stimulated ATP synthesis and phosphate transport in muscle of insulin-resistant offspring of type 2 diabetic parents. PLoS Med. 2, e233

38 Halvatsiotis, P. et al. (2002) Synthesis rate of muscle proteins, muscle functions, and amino acid kinetics in type 2 diabetes. Diabetes 51 , 2395-2404

39 Asmann, Y.W. et al. (2006) Skeletal muscle mitochondrial functions, mitochondrial DNA copy numbers, and gene transcript profiles in type 2 diabetic and nondiabetic subjects at equal levels of low or high insulin and euglycemia. Diabetes 55, 3309-3319

40 Brown, A.E. et al. (2008) Does impaired mitochondrial function affect insulin signaling and action in cultured human skeletal muscle cells? Am. J. Physiol. Endocrinol. Metab. 294, E97-E102

41 Nair, K.S. et al. (2008) Asian Indians have enhanced skeletal muscle mitochondrial capacity to produce ATP in association with severe insulin resistance. Diabetes 57, 1166-1175

42 Toledo, F.G. et al. (2008) Mitochondrial capacity in skeletal muscle is not stimulated by weight loss despite increases in insulin action and decreases in intramyocellular lipid content. Diabetes 57, 987-994

43 Schrauwen-Hinderling, V.B. et al. (2008) The insulin-sensitizing effect of rosiglitazone in type 2 diabetes mellitus patients does not require improved in vivo muscle mitochondrial function. J. Clin. Endocrinol. Metab. 93, 2917-2921

44 Pagel-Langenickel, I. et al. (2007) A discordance in rosiglitazone mediated insulin sensitization and skeletal muscle mitochondrial content/activity in type 2 diabetes mellitus. Am. J. Physiol. Heart Circ. Physiol. 293, H2659-H2666

45 Brons, C. et al. (2008) Mitochondrial function in skeletal muscle is normal and unrelated to insulin action in young men born with low birth weight. J. Clin. Endocrinol. Metab. 93, 3885-3892 
46 Eriksen, M.B. et al. (2011) Intact primary mitochondrial function in myotubes established from women with PCOS. J. Clin. Endocrinol. Metab. 96, E1298-E1302

47 Gallagher, I.J. et al. (2010) Integration of microRNA changes in vivo identifies novel molecular features of muscle insulin resistance in type 2 diabetes. Genome Med. 2, 9

48 Sleigh, A. et al. (2011) Mitochondrial dysfunction in patients with primary congenital insulin resistance. J. Clin. Invest. 121, 2457-2461

49 Nielsen, J. et al. (2010) Increased subsarcolemmal lipids in type 2 diabetes: effect of training on localization of lipids, mitochondria, and glycogen in sedentary human skeletal muscle. Am. J. Physiol. Endocrinol. Metab. 298, E706-E713

50 Phielix, E. et al. (2010) Exercise training increases mitochondria content and ex vivo mitochondrial function similarly in patients with type 2 diabetes and in control individuals. Diabetologia 53, 1714-1721

51 Hey-Mogensen, M. et al. (2010) Effect of physical training on mitochondrial respiration and reactive oxygen species release in skeletal muscle in patients with obesity and type 2 diabetes. Diabetologia 53, 1976-1985

52 Heilbronn, L.K. et al. (2006) Effect of 6-month calorie restriction on biomarkers of longevity, metabolic adaptation, and oxidative stress in overweight individuals: a randomized controlled trial. JAMA 295, 1539-1548
53 Larson-Meyer, D.E. et al. (2006) Effect of calorie restriction with or without exercise on insulin sensitivity, beta-cell function, fat cell size, and ectopic lipid in overweight subjects. Diabetes Care 29, 1337-1344

54 Larson-Meyer, D.E. et al. (2008) Effect of 6-month calorie restriction and exercise on serum and liver lipids and markers of liver function. Obesity (Silver Spring) 16, 1355-1362

55 Civitarese, A.E. et al. (2007) Calorie restriction increases muscle mitochondrial biogenesis in healthy humans. PLoS Med. 4, e76

56 Yamamoto, H. et al. (2007) Sirtuin functions in health and disease. Mol. Endocrinol. 21, 1745-1755

57 de Kreutzenberg, S.V. et al. (2010) Downregulation of the longevityassociated protein sirtuin 1 in insulin resistance and metabolic syndrome: potential biochemical mechanisms. Diabetes 59, 1006-1015

58 Rutanen, J. et al. (2010) SIRT1 mRNA expression may be associated with energy expenditure and insulin sensitivity. Diabetes 59, 829-835

59 Frojdo, S. et al. (2011) Phosphoinositide 3-kinase as a novel functional target for the regulation of the insulin signaling pathway by SIRT1. Mol. Cell. Endocrinol. 335, 166-176

60 Timmers, S. et al. (2011) Calorie restriction-like effects of 30 days of resveratrol supplementation on energy metabolism and metabolic profile in obese humans. Cell Metab. 14, 612-622

[61] Canto, C. et al. (2009) AMPK regulates energy expenditure by modulating NAD+ metabolism and SIRT1 activity. Nature 458, $1056-1060$ 